\title{
On Distance and Similarity Measures of Intuitionistic Fuzzy Multi Set
}

\author{
*P. Rajarajeswari, **N. Uma \\ * Department of Mathematics, Chikkanna Arts College, Tirupur, Tamil Nadu. (INDIA). \\ ** Department of Mathematics, SNR Sons College, Coimbatore, Tamil Nadu. (INDIA).
}

\begin{abstract}
In this paper, three distance measures and their corresponding similarity measures of Intuitionistic Fuzzy Multi sets (IFMS) are introduced and compared. The measures are based on Hausdroff distance measure, Geometric distance measure and the Normalized distance measure.
\end{abstract}

Key Words - Multi set, Intuitionistic fuzzy set, Intuitionistic Fuzzy Multi sets, Distance Measure, Similarity measure

\section{Introduction.}

The traditional Fuzzy sets (FS) introduced by Lofti A. Zadeh [1] in 1965 was the generalisation of Crisp sets presented by George Cantor. The fuzzy set allows the object to partially belong to a set with a membership degree $(\mu)$ between 0 and 1. Later, Krasssimir T. Atanassov [2, 3] proposed the Intuitionistic Fuzzy sets (IFS) as the generalisation of the Fuzzy set. The IFS represent the uncertainty with respect to both membership $(\mu \in[0,1])$ and non membership $(\vartheta \in[0,1])$ such that $\mu+\vartheta \leq 1$. The number $\pi=1-\mu-\vartheta$ is called the hesitiation degree or intuitionistic index. Using the distance and the similarity measures, the IFSs defined on the same universe are compared. And the study of distance and similarity measure of IFSs gives lots of measures, each representing specific properties and behaviour in real-life decision making and pattern recognition works.

Fuzzy Multi set $(F M S)$ concept was introduced by R. R. Yager [4]. Multi set [5] allows the repeated occurrences of any element and hence the Fuzzy Multi set can occur more than once with the possibly of the same or the different membership values. The new concept Intuitionistic Fuzzy Multi sets (IFMS) was proposed by T.K Shinoj and Sunil Jacob John [6].

This paper is an extension of the distance and similarity measure of IFMS. The numerical results of the examples show that the developed distance and similarity measures are well suited to use any linguistic variables.

The organization of this paper is as follows: In section 2, the Fuzzy Multi sets, Intuitionistic Fuzzy Multi sets and the distance and similarity measures of IFS are presented. The methodologies of three different distance and similarity measures are proposed for the IFMS in section 3. The section 4, analyses the numerical evaluation of the proposed methods.

Definition: 2.1

II. Preliminaries

Let $\mathrm{X}$ be a nonempty set. A fuzzy set $A$ in $X$ is given by $A=\left\{\left\langle x, \mu_{A}(x)\right\rangle / x \in X\right\}$

where $\mu_{A}: \mathrm{X} \rightarrow[0,1]$ is the membership function of the fuzzy set $A$ (i.e.) $\mu_{A}(x) \in[0,1]$ is the membership of $x \in X$ in $A$. The generalizations of fuzzy sets are the Intuitionistic fuzzy (IFS) set proposed by Atanassov $[1,2]$ is with independent memberships and non memberships.

\section{Definition: 2.2}

An Intuitionistic fuzzy set (IFS), $A$ in $X$ is given by $\mathrm{A}=\left\{\left\langle x, \mu_{A}(x), \vartheta_{A}(x)\right\rangle / x \in X\right\}$ where $\mu_{A}: \mathrm{X} \rightarrow[0,1]$ and $\vartheta_{A}: \mathrm{X} \rightarrow[0,1]$ with the condition $0 \leq \mu_{A}(x)+\vartheta_{A}(x) \leq 1, \forall x \in X$ Here $\mu_{A}(x)$ and $\vartheta_{A}(x) \in[0,1]$ denote the membership and the non membership functions of the fuzzy set $A$; For each Intuitionistic fuzzy set in $X, \pi_{A}(x)=1-\mu_{A}(x)-\left[1-\mu_{A}(x)\right]=0$ for all $x \in X$ that is $\pi_{A}(x)=1-\mu_{A}(x)-\vartheta_{A}(x)$ is the hesitancy degree of $x \in X$ in $A$. Always $0 \leq \pi_{A}(x) \leq 1, \forall x \in X$. The complementary set $A^{c}$ of $A$ is defined as $A^{c}=\left\{\left\langle x, \vartheta_{A}(x), \mu_{A}(x)\right\rangle / x \in X\right\}$

\section{Definition: 2.3}

Let $\mathrm{X}$ be a nonempty set. A Fuzzy Multi set (FMS) A in X is characterized by the count membership function Mc such that Mc $: X \rightarrow Q$ where $\mathrm{Q}$ is the set of all crisp multi sets in [0,1]. Hence, for any $x \in X, \operatorname{Mc}(\mathrm{x})$ is the crisp multi set from $[0,1]$. The membership sequence is defined as $\left(\mu_{A}^{1}(x), \mu_{A}^{2}(x), \ldots \ldots \ldots \mu_{A}^{p}(x)\right)$ where $\mu_{A}^{1}(x) \geq \mu_{A}^{2}(x) \geq \cdots \geq \mu_{A}^{p}(x)$. 
Therefore, A FMS $A$ is given by $A=\left\{\left\langle x,\left(\mu_{A}^{1}(x), \mu_{A}^{2}(x), \ldots \ldots \ldots \mu_{A}^{p}(x)\right)\right\rangle / x \in X\right\}$

\section{Definition: 2.4}

Let X be a nonempty set. A Intuitionistic Fuzzy Multi set (IFMS) A in X is characterized by two functions namely count membership function Mc and count non membership function NMc such that $\mathrm{Mc}: \mathrm{X} \rightarrow \mathrm{Q}$ and $\mathrm{NMc}: \mathrm{X} \rightarrow \mathrm{Q}$ where $\mathrm{Q}$ is the set of all crisp multi sets in $[0,1]$. Hence, for any $x \in X, \operatorname{Mc}(\mathrm{x})$ is the crisp multi set from $[0,1]$ whose membership sequence is defined as

$\left(\mu_{A}^{1}(x), \mu_{A}^{2}(x), \ldots \ldots \ldots \mu_{A}^{p}(x)\right)$ where $\mu_{A}^{1}(x) \geq \mu_{A}^{2}(x) \geq \cdots \geq \mu_{A}^{p}(x)$ and the corresponding non membership sequence $\mathrm{NMc}(\mathrm{x})$ is defined as $\left(\vartheta_{A}^{1}(x), \vartheta_{A}^{2}(x), \ldots \ldots \ldots \vartheta_{A}^{p}(x)\right)$ where the non membership can be either decreasing or increasing function. such that $0 \leq \mu_{A}^{i}(x)+\vartheta_{A}^{i}(x) \leq 1, \forall x \in X$ and $i=1,2, \ldots p$. Therefore, An IFMS $A$ is given by

$$
\begin{gathered}
A=\left\{\left\langle x,\left(\mu_{A}^{1}(x), \mu_{A}^{2}(x), \ldots \ldots \ldots \mu_{A}^{p}(x)\right),\left(\vartheta_{A}^{1}(x), \vartheta_{A}^{2}(x), \ldots \ldots \ldots \vartheta_{A}^{p}(x)\right)\right\rangle / x \in X\right\} \\
\text { where } \mu_{A}^{1}(x) \geq \mu_{A}^{2}(x) \geq \ldots \geq \mu_{A}^{p}(x)
\end{gathered}
$$

The complementary set $A^{c}$ of $A$ is defined as

$$
\begin{gathered}
A^{c}=\left\{\left\langle x,\left(\vartheta_{A}^{1}(x), \vartheta_{A}^{2}(x), \ldots \ldots \ldots \vartheta_{A}^{p}(x)\right),\left(\mu_{A}^{1}(x), \mu_{A}^{2}(x), \ldots \ldots \ldots \mu_{A}^{p}(x)\right),\right\rangle / x \in X\right\} \\
\text { where } \vartheta_{A}^{1}(x) \geq \vartheta_{A}^{2}(x) \geq \cdots \geq \vartheta_{A}^{p}(x)
\end{gathered}
$$

\section{Definition: 2.5}

The Cardinality of the membership function $\mathrm{Mc}(\mathrm{x})$ and the non membership function $\mathrm{NMc}(\mathrm{x})$ is the length of an element $\mathrm{x}$ in an IFMS A denoted as $\eta$, defined as $\eta=|\operatorname{Mc}(\mathrm{x})|=|\operatorname{NMc}(\mathrm{x})|$

If $\mathrm{A}, \mathrm{B}, \mathrm{C}$ are the IFMS defined on $X$, then their cardinality $\eta=\operatorname{Max}\{\eta(\mathrm{A}), \eta(\mathrm{B}), \eta(\mathrm{C})\}$.

\section{6: Distance Measures of Intuitionistic Fuzzy Sets}

In the IFS, the commonly defined distance measures $[\mathbf{7 , 8}]$ for sets $A, B$ in $X=\left\{x_{1}, x_{2}, \ldots \ldots x_{n}\right\}$ are

- In Hamming metrics, it is

$$
h_{d}(A, B)=\frac{1}{2} \sum_{i=1}^{n}\left(\left|\mu_{A}\left(x_{i}\right)-\mu_{B}\left(x_{i}\right)\right|+\left|\vartheta_{A}\left(x_{i}\right)-\vartheta_{B}\left(x_{i}\right)\right|\right)
$$

and with all three degrees taken under consideration, it is

$$
h_{d}(A, B)=\frac{1}{2} \sum_{i=1}^{n}\left(\left|\mu_{A}\left(x_{i}\right)-\mu_{B}\left(x_{i}\right)\right|+\left|\vartheta_{A}\left(x_{i}\right)-\vartheta_{B}\left(x_{i}\right)\right|+\left|\pi_{A}\left(x_{i}\right)-\pi_{B}\left(x_{i}\right)\right|\right)
$$

- In Hamming metrics, the Hausdroff distance is

$$
d_{h}(A, B)=\frac{1}{n} \sum_{i=1}^{n} \max \left\{\left(\left|\mu_{A}\left(x_{i}\right)-\mu_{B}\left(x_{i}\right)\right|,\left|\vartheta_{A}\left(x_{i}\right)-\vartheta_{B}\left(x_{i}\right)\right|\right)\right\}
$$

and with all three degrees taken under consideration, it is

$$
d_{h}(A, B)=\frac{1}{n} \sum_{i=1}^{n} \max \left\{\left(\left|\mu_{A}\left(x_{i}\right)-\mu_{B}\left(x_{i}\right)\right|,\left|\vartheta_{A}\left(x_{i}\right)-\vartheta_{B}\left(x_{i}\right)\right|,\left|\pi_{A}\left(x_{i}\right)-\pi_{B}\left(x_{i}\right)\right|\right)\right\}
$$

- $\quad$ The Geometric distance is

$$
D_{g}(A, B)=\sqrt{\left(\mu_{A}\left(x_{i}\right)-\mu_{B}\left(x_{i}\right)\right)^{2}+\left(\vartheta_{A}\left(x_{i}\right)-\vartheta_{B}\left(x_{i}\right)\right)^{2}}
$$

and with all degrees taken under consideration, is

$$
D_{g}(A, B)=\sqrt{\left(\mu_{A}\left(x_{i} x\right)-\mu_{B}\left(x_{i}\right)\right)^{2}+\left(\vartheta_{A}\left(x_{i}\right)-\vartheta_{B}\left(x_{i}\right)\right)^{2}+\left(\pi_{A}\left(x_{i}\right)-\pi_{B}\left(x_{i}\right)\right)^{2}}
$$

Hence the normalized Geometric distance is $D_{G}(A, B)=\frac{1}{\sqrt{2}} D_{g}(A, B)$

- $\quad$ The Normalized Hamming distance is

$$
h_{D}^{*}(A, B)=\frac{1}{2 n} \sum_{i=1}^{n}\left(\left|\mu_{A}\left(x_{i}\right)-\mu_{B}\left(x_{i}\right)\right|+\left|\vartheta_{A}\left(x_{i}\right)-\vartheta_{B}\left(x_{i}\right)\right|\right)
$$

and with all degrees taken under consideration is

$$
h_{D}^{*}(x, y)=\frac{1}{2 n} \sum_{i=1}^{n}\left(\left|\mu_{A}\left(x_{i}\right)-\mu_{B}\left(x_{i}\right)\right|+\left|\vartheta_{A}\left(x_{i}\right)-\vartheta_{B}\left(x_{i}\right)\right|+\left|\pi_{A}\left(x_{i}\right)-\pi_{B}\left(x_{i}\right)\right|\right)
$$

\section{7: Similarity Measures of Intuitionistic Fuzzy Sets}

\section{Definition: 2.7}

Let $S: X \times X \rightarrow[0,1]$ be a map. Then $S(A, B)$ is said to be the similarity measure between $A$ and $B$, where $A, B \in X$ and $X$ is and intuitionistic fuzzy set, if $S(A, B)$ satisfies the following properties
1. $\mathrm{S}(\mathrm{A}, \mathrm{B}) \in[0,1]$
2. $\mathrm{S}(\mathrm{A}, \mathrm{B})=1$ if and only if $\mathrm{A}=\mathrm{B}$
3. $S(A, B)=S(B, A)$

4. If $\mathrm{A} \subseteq \mathrm{B} \subseteq \mathrm{C} \in \mathrm{X}$, then $\mathrm{S}(\mathrm{A}, \mathrm{C}) \leq \mathrm{S}(\mathrm{A}, \mathrm{B}) \mathrm{S}(\mathrm{A}, \mathrm{C}) \leq \mathrm{S}(\mathrm{B}, \mathrm{C})$

5. $\mathrm{S}(\mathrm{A}, \mathrm{B})=0$ if and only if $\mathrm{A}=\varphi$ and $\mathrm{B}=\bar{A}$ (or) $\mathrm{A}=\bar{B}$ and $\mathrm{B}=\varphi$

The various similarity measures between Intuitionistic fuzzy sets have been defined during the past years. The most notable similarity measures which have been used in pattern recognition are the following.

- $\quad$ The Similarity measure proposed by Yanhong et al.[9] was $\boldsymbol{S}_{\boldsymbol{O}}(\boldsymbol{A}, \boldsymbol{B})$ 


$$
\boldsymbol{S}_{\boldsymbol{o}}(\boldsymbol{A}, \boldsymbol{B})=1-\sqrt{\frac{1}{2 n}\left(\sum_{i=i}^{n}\left(\mu_{A}\left(x_{i}\right)-\mu_{B}\left(x_{i}\right)\right)^{2}+\left(\vartheta_{A}\left(x_{i}\right)-\vartheta_{B}\left(x_{i}\right)\right)^{2}\right)}
$$

- $\quad$ Later Hung and Yang [10] presented their similarity measures based on Hausdorff distance as $S_{H Y}^{1}(A, B), \quad S_{H Y}^{2}(A, B), \quad S_{H Y}^{3}(A, B)$

$$
\begin{aligned}
& \boldsymbol{S}_{\boldsymbol{H} \boldsymbol{Y}}^{\mathbf{1}}(\boldsymbol{A}, \boldsymbol{B})=1-d_{H}(A, B) \\
& \boldsymbol{S}_{\boldsymbol{H} \boldsymbol{Y}}^{\mathbf{2}}(\boldsymbol{A}, \boldsymbol{B})=\left(e^{-d_{H}(A, B)}-e^{-1}\right) /\left(1-e^{-1}\right) \\
& \boldsymbol{S}_{\boldsymbol{H} \boldsymbol{Y}}^{\mathbf{3}}(\boldsymbol{A}, \boldsymbol{B})=\left(1-d_{H}(A, B)\right) /\left(1+d_{H}(A, B)\right)
\end{aligned}
$$

where $d_{H}(A, B)$ was the Hausdorff distance from equation (2.6.3 and 2.6.4)

- The similarity measure based on geometric distance was proposed by S. Sebastian, J. Philip [11] was

$$
\boldsymbol{S}_{\boldsymbol{G}}(\boldsymbol{A}, \boldsymbol{B})=1-D_{G}(A, B)
$$

- $\quad$ Using the concept of normalized Hamming distance, the similarity measure presented by

E. Szmidt, J. Kacprzyk, [12] was $\operatorname{Sim}(\boldsymbol{A}, \boldsymbol{B})=\frac{h_{D}^{*}(A, B)}{h_{D}^{*}\left(A, B^{c}\right)}$

\section{Proposed Distance And Similarity Measures For Intuitionistic Multi Fuzzy Sets}

In IFS, the distance and similarity measures are considered for the membership and non membership functions only once. But in IFMS, it should be considered more than once; because of their multi membership and non membership functions. And, their considerations are combined together by means of Summation concept based on their cardinality.

\section{1: HAUSDROFF MEASURE}

In Hamming metrics, the Hausdroff distance is defined as

$$
d_{h}(A, B)=\frac{1}{\eta} \sum_{j=1}^{\eta}\left\{\frac{1}{n} \sum_{i=1}^{n} \max \left[\left(\left|\mu_{A}^{j}\left(x_{i}\right)-\mu_{B}^{j}\left(x_{i}\right)\right|,\left|\vartheta_{A}^{j}\left(x_{i}\right)-\vartheta_{B}^{j}\left(x_{i}\right)\right|\right)\right]\right\}
$$

and with all three degrees, it is

$$
d_{h}(A, B)=\frac{1}{\eta} \sum_{j=1}^{\eta}\left\{\frac{1}{n} \sum_{i=1}^{n} \max \left[\left(\left|\mu_{A}^{j}\left(x_{i}\right)-\mu_{B}^{j}\left(x_{i}\right)\right|,\left|\vartheta_{A}^{j}\left(x_{i}\right)-\vartheta_{B}^{j}\left(x_{i}\right)\right|\right),\left|\pi_{A}^{j}\left(x_{i}\right)-\pi_{B}^{j}\left(x_{i}\right)\right|\right]\right\}
$$

Hence the Similarity measure based on the Hausdroff distance becomes

$$
\begin{aligned}
& \boldsymbol{S}_{\boldsymbol{H}}^{\mathbf{1}}(\boldsymbol{A}, \boldsymbol{B})=1-d_{h}(A, B) \\
& \boldsymbol{S}_{\boldsymbol{H}}^{\mathbf{2}}(\boldsymbol{A}, \boldsymbol{B})=\left(e^{-d_{h}(A, B)}-e^{-1}\right) /\left(1-e^{-1}\right) \\
& \boldsymbol{S}_{\boldsymbol{H}}^{\mathbf{3}}(\boldsymbol{A}, \boldsymbol{B})=\left(1-d_{h}(A, B)\right) /\left(1+d_{h}(A, B)\right)
\end{aligned}
$$

\section{2: GEOMETRIC MEASURE}

The Geometric distance of the Intuitionistic Multi Fuzzy set is defined as

$$
D_{g}(A, B)=\frac{1}{\eta} \sum_{j=1}^{\eta}\left\{\frac{1}{n} \sum_{i=1}^{n} \sqrt{\left(\mu_{A}^{j}\left(x_{i}\right)-\mu_{B}^{j}\left(x_{i}\right)\right)^{2}+\left(\vartheta_{A}^{j}\left(x_{i}\right)-\vartheta_{B}^{j}\left(x_{i}\right)\right)^{2}}\right\}
$$

and when all degrees are taken under consideration , it

$D_{g}(A, B)=\frac{1}{\eta} \sum_{j=1}^{\eta}\left\{\frac{1}{n} \sum_{i=1}^{n} \sqrt{\left(\mu_{A}^{j}\left(x_{i}\right)-\mu_{B}^{j}\left(x_{i}\right)\right)^{2}+\left(\vartheta_{A}^{j}\left(x_{i}\right)-\vartheta_{B}^{j}\left(x_{i}\right)\right)^{2}+\left(\pi_{A}^{j}\left(x_{i}\right)-\pi_{B}^{j}\left(x_{i}\right)\right)^{2}}\right\}$

Where the Normalized Geometric distance is $D_{G}(A, B)=\frac{1}{\sqrt{2}} D_{g}(A, B)$

Therefore the Similarity measure based on geometric distance is $\boldsymbol{S}_{\boldsymbol{G}}(\boldsymbol{A}, \boldsymbol{B})=1-D_{G}(A, B)$

\section{3: NORMALIZIED HAMMING MEASURE}

In the IFMS, the Normalized Hamming distance is

$$
N_{D}^{*}(A, B)=\frac{1}{\eta} \sum_{j=1}^{\eta}\left\{\frac{1}{2 n} \sum_{i=1}^{n}\left(\left|\mu_{A}^{j}\left(x_{i}\right)-\mu_{B}^{j}\left(x_{i}\right)\right|+\left|\vartheta_{A}^{j}\left(x_{i}\right)-\vartheta_{B}^{j}\left(x_{i}\right)\right|\right)\right\}
$$

and with all three degrees taken under consideration it becomes

$\left.N_{D}^{*}(A, B)=\frac{1}{\eta} \sum_{j=1}^{\eta}\left\{\frac{1}{2 n} \sum_{i=1}^{n}\left(\left|\mu_{A}^{j}\left(x_{i}\right)-\mu_{B}^{j}\left(x_{i}\right)\right|+\left|\vartheta_{A}^{j}\left(x_{i}\right)-\vartheta_{B}^{j}\left(x_{i}\right)\right|+\left|\pi_{A}^{j}\left(x_{i}\right)-\pi_{B}^{j}\left(x_{i}\right)\right|\right)\right\}\right\}$

Using the concept of Normalized Hamming distance, the Similarity measure in IFMS is 


$$
\operatorname{Sim}(\boldsymbol{A}, \boldsymbol{B})=\frac{N_{D}^{*}(A, B)}{N_{D}^{*}\left(A, B^{c}\right)}
$$

Where $B^{c}$ is the complement set of $B$ such that

$B^{c}=\left\{\left\langle x,\left(\vartheta_{A}^{1}(x), \vartheta_{A}^{2}(x), \ldots \ldots \ldots \vartheta_{A}^{p}(x)\right),\left(\mu_{A}^{1}(x), \mu_{A}^{2}(x), \ldots \ldots \ldots \mu_{A}^{p}(x)\right)\right\rangle / x \in X\right\}$, where $\vartheta_{A}^{1}(x) \geq \vartheta_{A}^{2}(x) \geq \cdots \geq \vartheta_{A}^{p}(x)$.

\section{Numerical Evaluation}

\section{EXAMPLE: 4.1}

Let $X=\left\{A_{1}, A_{2}, A_{3}, A_{4}, A_{5} \ldots A_{n}\right\}$ with $A=\left\{A_{1}, A_{2}, A_{3}, A_{4}, A_{5}\right\}$ and $B=\left\{A_{6}, A_{7}, A_{8}, A_{9}, A_{10}\right\}$ such that the IFMS $\mathrm{A}$ and $\mathrm{B}$ are defined in terms of membership and non-members

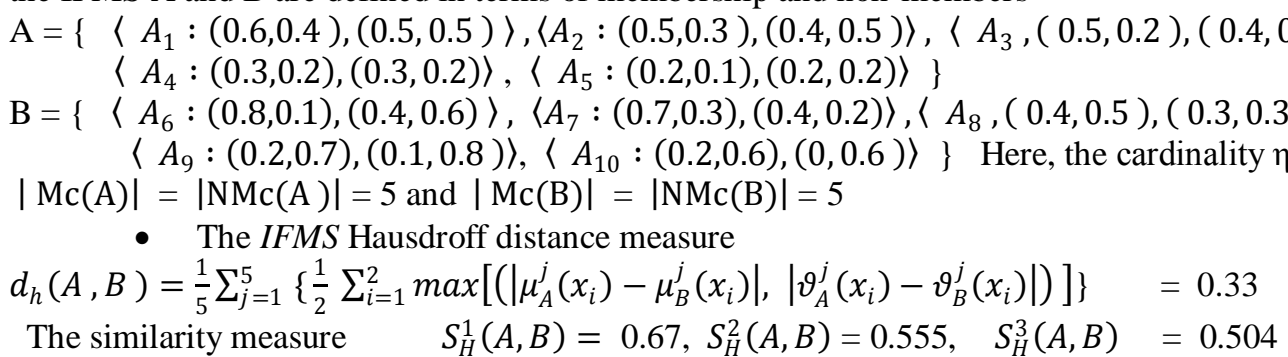

- The IFMS Geometric distance measure is

$$
\begin{aligned}
D_{g}(A, B) & =\frac{1}{5} \sum_{j=1}^{5}\left\{\frac{1}{2} \sum_{i=1}^{2} \sqrt{\left(\mu_{A}^{j}\left(x_{i}\right)-\mu_{B}^{j}\left(x_{i}\right)\right)^{2}+\left(\vartheta_{A}^{j}\left(x_{i}\right)-\vartheta_{B}^{j}\left(x_{i}\right)\right)^{2}}\right\}=0.365 \\
D_{G}(A, B) & =0.25887 . \text { Therefore the Similarity measure } S_{G}(A, B)=0.74113 \\
\bullet \quad \text { In the IFMS, the Normalized Hamming distance is } &
\end{aligned}
$$

$N_{D}^{*}(A, B)=\frac{1}{5} \sum_{j=1}^{5}\left\{\frac{1}{2 * 2} \sum_{i=1}^{2}\left(\left|\mu_{A}^{j}\left(x_{i}\right)-\mu_{B}^{j}\left(x_{i}\right)\right|+\left|\vartheta_{A}^{j}\left(x_{i}\right)-\vartheta_{B}^{j}\left(x_{i}\right)\right|\right)\right\}$

Hence using Normalized Hamming distance, the Similarity measure $\operatorname{Sim}(A, B)=\frac{0.145}{0.185}=0.78378$

\section{EXAMPLE: 4.2}

Let $\mathrm{X}=\left\{\mathrm{A}_{1}, \mathrm{~A}_{2}, \mathrm{~A}_{3}, \mathrm{~A}_{4} \ldots \ldots . \mathrm{A}_{\mathrm{n}}\right\}$ with $\mathrm{A}=\left\{\mathrm{A}_{1}, \mathrm{~A}_{2}\right.$, $\}$ and $\mathrm{B}=\left\{\mathrm{A}_{3}, \mathrm{~A}_{4}\right\}$ are the IFMS $\mathrm{A}$ and $\mathrm{B}$ defined as $\mathrm{A}=\left\{\left\langle A_{1}:(0.4,0.2,0.1),(0.3,0.1,0.2),(0.2,0.1,0.2),(0.1,0.4,0.3)\right\rangle\right.$,

$$
\left.\left\langle A_{2}:(0.6,0.3,0),(0.4,0.5,0.1),(0.4,0.3,0.2),(0.2,0.6,0.2)\right\rangle\right\}
$$

$\mathrm{B}=\left\{\left\langle A_{3}:(0.5,0.2,0.3),(0.4,0.2,0.3),(0.4,0.1,0.2),(0.1,0.1,0.6)\right\rangle\right.$

$\left.\left\langle A_{4}:(0.4,0.6,0.2),(0.4,0.5,0),(0.3,0.4,0.2),(0.2,0.4,0.1)\right\rangle\right\}$

The cardinality $\eta=2$ as $\quad|\operatorname{Mc}(\mathrm{A})|=|\mathrm{NMc}(\mathrm{A})|=2$ and $|\mathrm{Mc}(\mathrm{B})|=|\mathrm{NMc}(\mathrm{B})|=2$

- The IFMS Hausdroff distance measure

$d_{h}(A, B)=\frac{1}{2} \sum_{j=1}^{2}\left\{\frac{1}{4} \sum_{i=1}^{4} \max \left[\left(\left|\mu_{A}^{j}\left(x_{i}\right)-\mu_{B}^{j}\left(x_{i}\right)\right|,\left|\vartheta_{A}^{j}\left(x_{i}\right)-\vartheta_{B}^{j}\left(x_{i}\right)\right|\right),\left|\pi_{A}^{j}\left(x_{i}\right)-\pi_{B}^{j}\left(x_{i}\right)\right|\right]\right\}=0.1875$

The similarity measure $S_{H}^{1}(A, B)=0.8125, S_{H}^{2}(A, B)=0.7295, S_{H}^{3}(A, B)=0.684$

- $\quad$ The IFMS Geometric distance measure is

$D_{g}(A, B)=\frac{1}{2} \sum_{j=1}^{2}\left\{\frac{1}{4} \sum_{i=1}^{4} \sqrt{\left(\mu_{A}^{j}\left(x_{i}\right)-\mu_{B}^{j}\left(x_{i}\right)\right)^{2}+\left(\vartheta_{A}^{j}\left(x_{i}\right)-\vartheta_{B}^{j}\left(x_{i}\right)\right)^{2}+\left(\pi_{A}^{j}\left(x_{i}\right)-\pi_{B}^{j}\left(x_{i}\right)\right)^{2}}\right\}=0.23725$

$D_{G}(A, B)==0.1683$. Therefore the Similarity measure based on Geometric distance $S_{G}(A, B)=0.8317$

- In the IFMS, the Normalized Hamming distance is

$\left.N_{D}^{*}(A, B)=\frac{1}{2} \sum_{j=1}^{2}\left\{\frac{1}{2 * 4} \sum_{i=1}^{4}\left(\left|\mu_{A}^{j}\left(x_{i}\right)-\mu_{B}^{j}\left(x_{i}\right)\right|+\left|\vartheta_{A}^{j}\left(x_{i}\right)-\vartheta_{B}^{j}\left(x_{i}\right)\right|+\left|\pi_{A}^{j}\left(x_{i}\right)-\pi_{B}^{j}\left(x_{i}\right)\right|\right)\right\}\right\}$

Hence using Normalized Hamming distance, the Similarity measure is $\operatorname{Sim}(A, B)=\frac{0.16875}{0.21875}=0.77143$

\section{EXAMPLE: 4.3}

Let $X=\left\{A_{1}, A_{2}, A_{3}, A_{4} \ldots . A_{n}\right\}$ with $A=\left\{A_{1}, A_{2}, A_{3}\right\}$ and $B=\left\{A_{6}\right\}$ such that the IFMS $A$ and $B$ are

$\mathrm{A}=\left\{\left\langle A_{1}:(0.6,0.2,0.2),(0.4,0.3,0.3),(0.1,0.7,0.2),(0.5,0.4,0.1),(0.2,0.6,0.2)\right\}\right.$,

$\left\langle A_{2}:(0.7,0.1,0.2),(0.3,0.6,0.1),(0.2,0.7,0.1),(0.6,0.3,0.1),(0.3,0.4,0.3)\right\rangle$

$\left.\left\langle A_{3}:(0.5,0.4,0.1),(0.4,0.4,0.2),(0,0.8,0.2),(0.7,0.2,0.1),(0.4,0.4,0.2)\right\rangle\right\}$

$\mathrm{B}=\left\{\left\langle A_{6}:(0.8,0.1,0.1),(0.2,0.7,0.1),(0.3,0.5,0.2),(0.5,0.3,0.2),(0.5,0.4,0.1)\right\rangle\right\}$

Here $\mathrm{L}(\mathrm{A}, \mathrm{B})=\eta=3$ as $\quad|\mathrm{Mc}(\mathrm{A})|=|\mathrm{NMc}(\mathrm{A})|=3$ and $|\operatorname{Mc}(\mathrm{B})|=|\mathrm{NMc}(\mathrm{B})|=1$

Hence, their cardinality $\eta=\operatorname{Max}\{\eta(\mathrm{A}), \eta(\mathrm{B})\}=\max \{3,1\}=3$.

- The IFMS Hausdroff distance measure

$d_{h}(A, B)=\frac{1}{3} \sum_{j=1}^{3}\left\{\frac{1}{5} \sum_{i=1}^{n} \max \left[\left(\left|\mu_{A}^{j}\left(x_{i}\right)-\mu_{B}^{j}\left(x_{i}\right)\right|,\left|\vartheta_{A}^{j}\left(x_{i}\right)-\vartheta_{B}^{j}\left(x_{i}\right)\right|\right),\left|\pi_{A}^{j}\left(x_{i}\right)-\pi_{B}^{j}\left(x_{i}\right)\right|\right]\right\}=0.20667$

The similarity measure $S_{H}^{1}(A, B)=0.79333, S_{H}^{2}(A, B)=0.7046, S_{H}^{3}(A, B)=0.6575$ 
- The IFMS Geometric distance measure becomes

$D_{g}(A, B)=\frac{1}{3} \sum_{j=1}^{3}\left\{\frac{1}{5} \sum_{i=1}^{5} \sqrt{\left(\mu_{A}^{j}\left(x_{i}\right)-\mu_{B}^{j}\left(x_{i}\right)\right)^{2}+\left(\vartheta_{A}^{j}\left(x_{i}\right)-\vartheta_{B}^{j}\left(x_{i}\right)\right)^{2}+\left(\pi_{A}^{j}\left(x_{i}\right)-\pi_{B}^{j}\left(x_{i}\right)\right)^{2}}\right\}=0.2734$

$D_{G}(A, B)=0.1939$. Therefore the Similarity measure based on geometric distance $S_{G}(A, B)=0.8061$

- In the IFMS, the Normalized Hamming distance is

$\left.N_{D}^{*}(A, B)=\frac{1}{3} \sum_{j=1}^{3}\left\{\frac{1}{2 * 5} \sum_{i=1}^{5}\left(\left|\mu_{A}^{j}\left(x_{i}\right)-\mu_{B}^{j}\left(x_{i}\right)\right|+\left|\vartheta_{A}^{j}\left(x_{i}\right)-\vartheta_{B}^{j}\left(x_{i}\right)\right|+\left|\pi_{A}^{j}\left(x_{i}\right)-\pi_{B}^{j}\left(x_{i}\right)\right|\right)\right\}\right\}$

Hence using Normalized Hamming distance, the Similarity measure is $\operatorname{Sim}(A, B)=\frac{0.21667}{0.36}=0.60186$

From the examples 4.1 and 4.2 it is clear that the new measures perform well in the case of two representatives of IFMS (membership, non- membership function) and three representatives of IFMS (membership, nonmembership and hesitation function). The example 4.3 depicts the effective measure to check the distance and similarity between the IFMS and IFS.

\section{Conclusion}

Three methods of distance and similarity measure of IFMS are presented and analyzed. The proposed methods are mathematically valid and can be applied to any decision making problems or pattern recognitions. From the numerical evaluation is clear that the proposed similarity measure also satisfy the condition and properties of similarity measure (Definition: 2.7). The unique feature of this proposed method is that it considers multi membership and non membership for the same element. The present study constitutes a first study of distance measures based on IFMS and future research will establish the proposed methodology as a concrete pattern classification framework.

\section{References}

[1] Zadeh L. A., Fuzzy sets, Information and Control 8 (1965) 338-353.

[2] Atanassov K., Intuitionistic fuzzy sets, Fuzzy Sets and System 20 (1986) 87-96.

[3] Atanassov K., More on Intuitionistic fuzzy sets, Fuzzy Sets and Systems 33 (1989) 37-46.

[4] Yager R. R., On the theory of bags,(Multi sets), Int. Jou. Of General System, 13 (1986) 23-37.

[5] Blizard W. D., Multi set Theory, Notre Dame Journal of Formal Logic, Vol. 30, No. 1 36-66, (1989).

[6] Shinoj T.K., Sunil Jacob John, Intuitionistic Fuzzy Multi sets and its Application in Medical Diagnosis, World Academy of Science, Engineering and Technology, Vol. 61 (2012).

[7] Szmidt E., Kacprzyk J., On measuring distances between Intuitionistic fuzzy sets, Notes on IFS, Vol. 3 (1997) 1- 13.

[8] Szmidt E., Kacprzyk J., Distances between Intuitionistic fuzzy sets. Fuzzy Sets System, 114 (2000) 505-518.

[9] Yanhong L., Olson D., Qin Z., Similarity Measures between Intuitionistic Fuzzy sets : A Comparative Analysis, Pattern Recognition Letters, 28(2) (2007) 278 - 285.

[10] Hung W.L., Yang M. S. , Similarity Measures of Intuitionistic Fuzzy Sets Based on Hausdorff Distance, Pattern Recognition Letters, 25 (2004) 1603-1611.

[11] Souriar Sebastian and James Philip, Similarity Measure for Intuitionistic Fuzzy sets : An Intuitive Approach, Journal of Comp. \& Math. Sci. Vol. (1) (2012) 63-69.

[12] Szmidt E., Kacprzyk J., A similarity measure for Intuitionistic fuzzy sets and its application in supporting medical diagnostic reasoning. ICAISC 2004, Vol. LNAI 3070 (2004) 388-393.

[13] Mitchell H., On the Defgfeng - Chuntian Similarity Measure and its Application to Pattern Recognition, Pattern Recognition Letters, 24 (2003) 3101- 3104. 